\title{
Photon-Assisted Shot Noise in Normal Metal-Insulator-Superconductor Heterostructures
}

\author{
I. V. Boylo \\ Donetsk Institute for Physics and Engineering, Donetsk, Ukraine
}

Email address:

boylo@fti.dn.ua

To cite this article:

I. V. Boylo. Photon-Assisted Shot Noise in Normal Metal-Insulator-Superconductor Heterostructures. Journal of Photonic Materials and Technology. Vol. 1, No. 1, 2015, pp. 15-18. doi: 10.11648/j.jmpt.20150101.13

\begin{abstract}
Photon-assisted electron transport in mesoscopic heterostructures is studied via a scattering approach. The nonequilibrium current fluctuations of normal metal-insulator-superconductor systems under illumination with monochromatic microwaves have been calculated. Using a time-periodic potential applied across the tunnel junction we model the emission and absorption of photons. We have concluded that the photon-assisted shot noise decreases when the insulating barrier becomes more transparent. The voltage dependence of the shot-noise power as a function of microwave frequency has been investigated as well.
\end{abstract}

Keywords: Photon-Assisted Electron Transport, Shot Noise, Mesoscopic Heterostructure

\section{Introduction}

We study the low-frequency nonequilibrium current fluctuations generated by traveling of photon excited electrons and holes which are scattered by a conductor as a probe of mesoscopic heterostructures based on a superconducting metal. We are interested in how these influences the dc junction current fluctuations $\mathrm{S}$. It is known that electrical noise in mesoscopic systems can be characterized by the correlation function $S\left(t-t^{\prime}\right)=\left\langle\Delta I(t) \Delta I\left(t^{\prime}\right)\right\rangle$ of the electrical current I or its Fourier transform $S(V, \omega)$. Shot noise Sshot in a normal metal-insulator-superconductor junction as a consequence of the quantization of the charge appears when electrons are incident upon a tunnel barrier.

In this paper, we study the zero-frequency shot-noise power $S_{\text {shot }}(V, \omega \rightarrow 0)$ in a transport state ( $\mathrm{V}$ is the voltage bias). Investigations of the nonequilibrium current fluctuations can reveal information about the electron transport which is not present in the conductance or the electrical current vs voltage curve. For instance, we can extract the value of the elementary charge from the shot-noise power as was proposed by Schottky. The voltage dependence of the ratio of the actual shot noise $\mathrm{S}(\mathrm{V})$ and the Poisson noise $S_{\mathrm{P}}(V)$, called the Fano factor $F(V)=S(V) / S_{\mathrm{P}}(V)$, measures the unit of transferred charge.

The scattering-matrix approach to phase-coherent electron transport was generalized to the case of photon-assisted tunneling in a normal metal $[1,2]$. Excess noise in the coherent conductor with two contacts which is bent into connected loop in the presence of constant voltage and alternating external field was investigated by Lesovik and Levitov [1]. Photon-assisted transport was studied experimentally by Schoelkopf et al. [3], and many others [4, 5]. The shot-noise features at voltages corresponding to the photon energies were observed [3].

There have been papers of that kind learning the shot noise in ac biased junctions for many years. A lot of authors became interested in the subject towards the end of the last decades. For example, photon-assisted tunneling with nonclassical light in a superconducting microwave cavity coupled to a conductor in the tunneling regime has been recently investigated [6]. A non-trivial behavior of the current with the frequency and amplitude of the external ac field has been analyzed [7]. The heat current flowing into the superconductor ac voltage biased is found reduced in the photon-assisted tunnel regime [8]. Our goal will be to understand how microwaves affect the nonequilibrium current fluctuations in normal metal-insulator-superconductor contacts. As will be seen later on, the influence of photon-assisted tunneling, as compared to the noise power in contacts biased with dc voltage, is to increase the magnitude of the nonequilibrium current fluctuations $\mathrm{S}(\mathrm{V})$.

\section{Model}

In the paper, we follow an idea proposed in [2] to study the zero-frequency photon-assisted shot noise S. The model [9] 
we need to calculate the nonequilibrium current fluctuations in the superconducting heterostructures has been developed within the framework of scattering theory [10] and let us to examine photon-assisted electron transport in a normal metal-insulator-superconductor (NIS) junction.

\subsection{General Expression for Shot Noise in the Frame of Scattering Theory Applied To Superconducting Heterostructures}

Our system under investigation is a NIS tunnel contact, with

$$
\begin{aligned}
S_{\text {shot }}(V) & =\frac{4 e^{2}}{h} \int_{-\infty}^{\infty}\left\{\left[1-\left|R^{\mathrm{ee}}(\varepsilon)\right|^{2}\right] \cdot\left|R^{\mathrm{ee}}(\varepsilon)\right|^{2}+\left|R^{\mathrm{eh}}(\varepsilon)\right|^{2} \cdot\left[1-\left|R^{\mathrm{eh}}(\varepsilon)\right|^{2}\right]+\right. \\
& \left.+2 \cdot\left|R^{\mathrm{ee}}(\varepsilon)\right|^{2} \cdot\left|R^{\mathrm{eh}}(\varepsilon)\right|^{2}\right\} \cdot\left(f_{\mathrm{L}}(\varepsilon-e V)-f_{\mathrm{R}}(\varepsilon)\right)^{2} d \varepsilon
\end{aligned}
$$

where $\mathrm{h}$ is the Planck constant, e is the electrical charge, $\varepsilon$ is the electron energy relative to Fermi energy, the Fermi distribution in each reservoir $f(\varepsilon)=\left[1+\exp \left(\varepsilon / k_{\mathrm{B}} T\right)\right]^{-1}, \mathrm{~T}$ is the temperature of the electrons in the contacts. $\operatorname{Ree}(\varepsilon)$ is the total probability amplitude of electron-electron reflections and $\operatorname{Reh}(\varepsilon)$ is the total probability amplitude of electron-hole a normal metal $(\mathrm{N})$, a superconducting lead (S), and a dielectric barrier I between two metal electrodes. We start with the scattering approach [10] and consider a mesoscopic sample connected to two reservoirs, left (L) and right (R), when one of the leads is in the superconducting state. The zero-frequency correlation function $\operatorname{Sshot}(\mathrm{V})$ in a two-terminal superconducting heterostructure is expressed through the Fermi functions, fL and fR, and given by the formula

transformations. The summation was carried out on all possible charge paths i including Andreev transformations, carriers as having spin up and spin down s.

The energy dependence of the transmission coefficients lies in the scattering characteristics for an electron reflected into a hole and vice versa [11]

$$
r^{e h(h e)}(\Theta)=\frac{(\varepsilon+i \delta)-\operatorname{sign}(\varepsilon) \sqrt{(\varepsilon+i \delta)^{2}-\Delta^{2}(\Theta)}}{|\Delta(\Theta)|} \exp (\mp i \Phi(\Theta))
$$

where $\delta$ is the Dynes parameter, we will use $\delta=10-3$, and the angular dependence of the order parameter phase $\Phi(\Theta)$ and module $\Delta(\Theta)$ are determined by the symmetry of the superconducting state ( $\mathrm{s}$ - or d-wave type). In the most cases (s-wave symmetry), the dependence on the tunneling direction $\Theta$ is weak and $|\Delta(\Theta)|=\Delta_{s}$. In the Nb-based tunnel junction, the superconducting gap $\Delta \mathrm{s}$ follows the BCS relation $2 \Delta 0=3.5 \mathrm{kBTc}$.

\subsection{Modelling of Photon-Assisted Shot Noise in Superconducting Heterostructures}

Photon-assisted tunneling is known to be equivalent to simply having an ac bias voltage across a conductor. Thus, photon-assisted electron transport through the normal metal-insulator-superconductor (NIS) structure can be modeled using potential that consists of two parts: a static (dc) one $\mathrm{eV}$, and a dynamical (ac) one, which depends periodically

$$
\Psi_{n}(x, t, \varepsilon)=\varphi_{n}(x, \varepsilon) \exp (-i 2 \pi \varepsilon t / h) \sum_{l=-\infty}^{\infty} J_{l}\left(\frac{e V(v)}{h v}\right) \exp (-i l 2 \pi v t)
$$

where $\varphi_{n}(x, \varepsilon)$ is the wave function describing an electron in the left contact in channel $\mathrm{n}$ in the absence of a modulation potential $\mathrm{eV}(v), 1$ is an integer, $J_{l}\left(\frac{e V(v)}{h v}\right)$ is the lth - order

zero-frequency component of Sshot(V) to the case when the applied voltage is time-dependent. In this case, the relation between the shot-noise power $\mathrm{S}$ and the dc bias $\mathrm{V}$ becomes more complicated:

Bessel function.

Now we generalize the expression (1) for the

$$
\begin{aligned}
& S_{\text {shot }}(V)=\frac{4 e^{2}}{h} \int_{-\infty}^{\infty} \sum_{l=-\infty}^{\infty}\left(J_{l}\left(\frac{e V(v)}{h v}\right)\right)^{2}\left\{\left[1-\left|R^{\mathrm{ee}}(\varepsilon)\right|^{2}\right] \cdot\left|R^{\mathrm{ee}}(\varepsilon)\right|^{2}+\left|R^{\mathrm{eh}}(\varepsilon)\right|^{2} \cdot\left[1-\left|R^{\mathrm{eh}}(\varepsilon)\right|^{2}\right]+\right. \\
& \left.+2 \cdot\left|R^{\mathrm{ee}}(\varepsilon)\right|^{2} \cdot\left|R^{\mathrm{eh}}(\varepsilon)\right|^{2}\right\} \cdot(f(\varepsilon-e V+l h v)-f(\varepsilon))^{2} d \varepsilon
\end{aligned}
$$


where the time-independent $\mathrm{V}$ can be taken real valued, the maximum amplitude $\mathrm{V}(\mathrm{v})$ is some function of the frequency of applied microwaves $v$ and it can be tuned.

In the absence of an ac potential $(v \rightarrow 0)$ we have (1).

\section{Results and Discussion}

Let us now present our numerical results. Using the sum of Bessel functions in (4) we calculate numerically the voltage dependence of the shot-noise power in the frequency range from 0.5 to $5 \mathrm{GHz}$ at the temperature of liquid helium $(\mathrm{T}=4.2$ Kelvin).

\subsection{Photon-Assisted Shot Noise in the Tunneling Limit with a Small Barrier Transparency}

In the tunneling limit with $\mathrm{D}<<1$ performing the energy integration we obtain the nonequilibrium current fluctuations for the $\mathrm{Nb}$-based junctions (Fig.1).

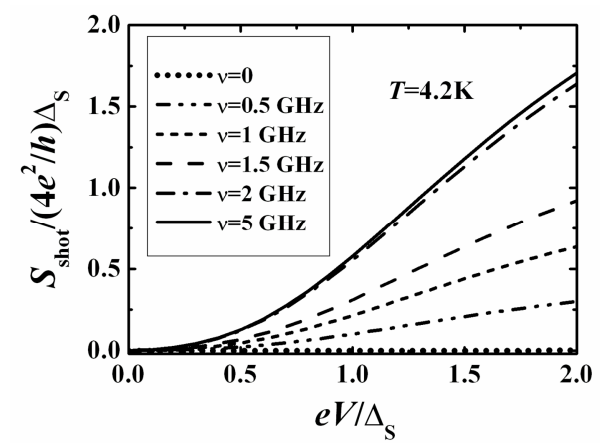

Figure 1. Electrical noise power Sshot in units of $(4 e 2 / h) \Delta S$ as a function of the constant voltage V for NIS tunnel structures with the barrier transparency $D=0.001$ at liquid-helium temperature $(T=4.2 \mathrm{~K})$. The frequency $v$ is fixed as $0.5,1,1.5,2$ and $5 \mathrm{GHz}$. Dotted line shows behavior without ac excitation $(v=0)$.

We note that the noise power $\mathrm{S}$ increases with the voltage bias $\mathrm{V}$ for all values of frequency. For a comparison, dotted line in Fig. 1 reproduce the zero-frequency expression, taking into account that in this case only the Bessel function with $1=0$ survives and should be taken equal to one. The noise power $\mathrm{S}(\mathrm{V})$ is always larger than $v=0$ characteristic. This means that photon-assisted electron transport gives rise to an enhancement of the nonequilibrium current fluctuations.

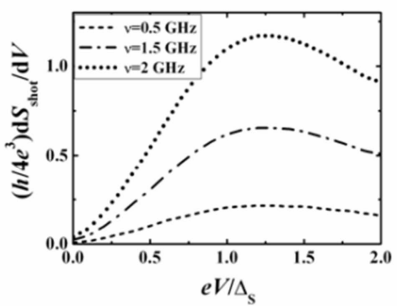

(a)

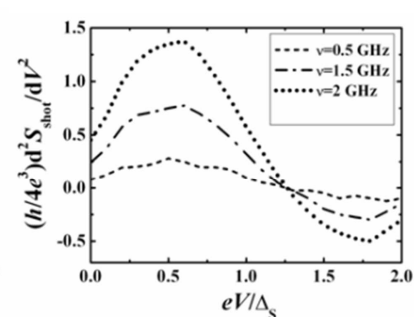

(b)
Figure 2. Differential shot noise versus voltage for NIS contacts in tunnel limit with 0.5, 1.5, and $2 \mathrm{GHz}$ ac excitations at $4.2 \mathrm{~K}$. (a) Dependence of $d S / d V$. (b) Second derivative of the shot noise (d2S/dV2).
It is possible to find the derivative of the sample's noise with voltage. In Fig. 2 the first and second derivatives are plotted as a function of the applied dc voltage $\mathrm{V}$.

The derivative $\mathrm{dS} / \mathrm{dV}$ increases up to the maximum and thereafter decreases. The superconductor with a conventional s-wave symmetry of the pair potential provides a well-known singularity at $\mathrm{eV}=\Delta \mathrm{s}$, where $\Delta \mathrm{s}$ is the related gap value. The peak of the first derivative of the shot-noise power near the energy of the superconducting gap is sharper with high frequency. The position of the maxima is slightly shifted from $\mathrm{eV}=\Delta \mathrm{s}$ toward higher values of $\mathrm{V}$. Note that the second derivative changes sign in the frequency range considered. As a result, the shot-noise power with positive $\mathrm{d} 2 \mathrm{~S} / \mathrm{dV} 2$ curves upwards, while the shot-noise power with negative one curves downwards.

\subsection{Photon-Assisted Shot Noise in NIS Structures with Various Transparencies of Insulating Barrier}

We have calculated the voltage dependence of the nonequilibrium current fluctuations $\mathrm{S}(\mathrm{V})$ for various insulating barrier transparencies D at $4.2 \mathrm{~K}$ (Fig. 3).

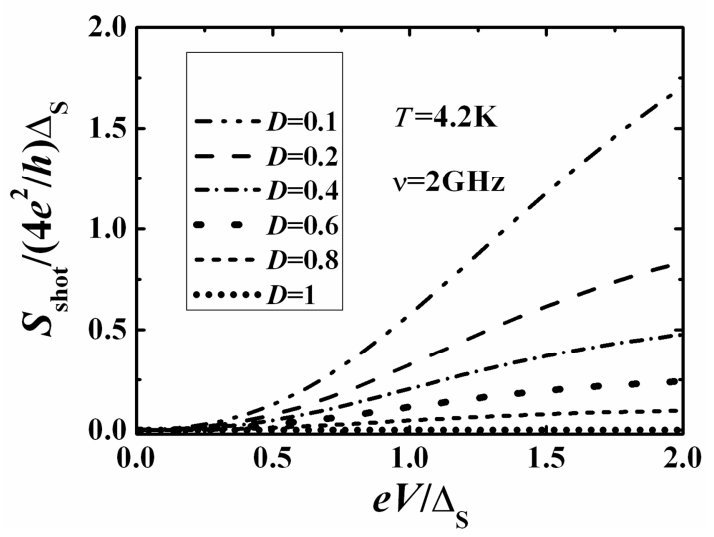

Figure 3. Voltage dependence of the shot noise power $S$ in units of $(4 e 2 / h) \Delta S$ for NIS tunnel structures as a function of the barrier transparency $D$ for microwave frequency $v=2 \mathrm{GHz}$ at $T=4.2$.

In Fig. 3 the various curves refer to different values of $\mathrm{D}$ at frequency $v=2 \mathrm{GHz}$.

We find that the nonequilibrium current fluctuations monotonically increase by increasing dc voltage bias $\mathrm{V}$. The spectral density of the shot noise Sshot depends on the scattering amplitudes in such a way that it decreases when the barrier becomes more transparent. For open channels $D=0$ shot noise disappears. In the limit that the barrier transparency $\mathrm{D}$ is very small, the shot-noise power corresponds to the uncorrelated photon-assisted tunneling of electrons.

In the case of $h v<<\Delta$, if the ac frequency is small compared to the superconducting gap corresponding to the value of $688 \mathrm{GHz}$ [12] which would lead to breaking up of the Cooper pairs we obtain the shot noise vs frequency characteristics plotted in Fig.4. By tuning the frequency $v$, one tunes the energy of photons hv. Calculations were performed up to $v=3 \mathrm{GHz}$. It is obvious that increasing the energy of 
photons, the shot-noise power becomes stronger.

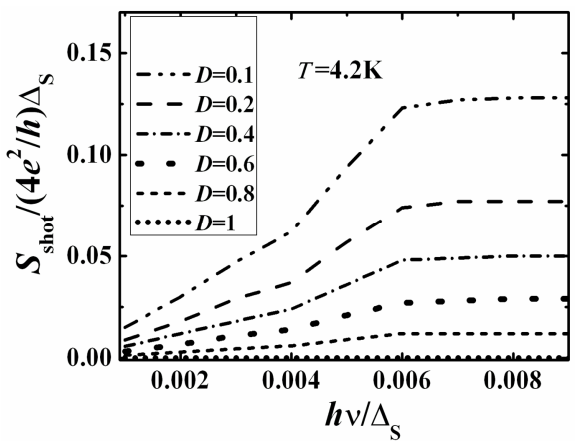

Figure 4. Interdependence between the shot noise power $S$ in units of $(4 e 2 / h) \Delta S$ and the energy of photons $h v$ for NIS tunnel structures with defferent values of the barrier transparency $D$ at $T=4.2 \mathrm{~K}$; the time-independent potential $\mathrm{eV}=0.5 \Delta S, \Delta S=1.47 \mathrm{meV}$.

\section{Conclusions}

In this paper, theoretical study of photon-assisted shot noise in normal metal-insulator-superconductor junctions has been performed. Modeling is used to explain and predict the behavior of shot noise in the Nb-based heterostructures under the microwave illumination. Following the findings of the work [10], the shot-noise power in tunnel contacts based on s-wave superconductors has been considered. We have discussed how the nonequilibrium current fluctuations depend on various parameters. Voltage dependence of shot noise $\mathrm{S}(\mathrm{V})$ for tunnel contacts with different insulating barriers has been calculated at the temperature of liquid helium.

To summarize, we conclude that photon-assisted shot noise is always increased as compared to its magnitude in contacts biased with dc voltage. The approach described above will be useful in future studies to model and predict the behavior of the nonequilibrium current fluctuations in normal metal-insulator-superconductor junctions.

\section{References}

[1] G. B. Lesovic and L. S. Levitov, "Noise in an ac Biased Junction: Nonstationary Aharonow-Bohm Effect", Phys. Rev. Lett., vol. 72, pp. 538-541, January 1994.
[2] M. H. Pedersen, M. Büttiker, "Scattering theory of photon-assisted electron transport", Phys. Rev. B, vol. 58, pp. 12993-13006, November 1998.

[3] R. J. Schoelkopf, A. A. Kozhevnikov, and D. E. Prober, "Observation of "Photon-Assisted" Shot Noise in a Phase-Coherent Conductor", Phys. Rev. Lett., vol. 80, pp. 2437-2440, March 1998.

[4] J. Gabelli, B. Reulet,"Dynamics of quantum noise in a tunnel junction under ac excitation", Phys. Rev. Lett., vol. 100, pp. 026601-1 - 026601-4, January 2008.

[5] J. Basset, H. Bouchiat, and R. Deblock, "Emission and absorption quantum noise measurement with an on-chip resonant circuit”, Phys. Rev. Lett., vol. 105, pp. 166801-1 166801-4, October 2010.

[6] J.-R. Souquet, M. J. Woolley, J. Gabelli, P. Simon and A. A. Clerk, "Photon- assisted tunnelling with nonclassical light", Nature Communications, vol. 5, pp. 5562-1 - 5562-9, November 2014.

[7] F. Gallego-Marcos, R. Sánchez and G. Platero, "Photon assisted long-range tunneling", J. Appl. Phys., vol. 117, pp. 112808-1 - 112808-7, March 2015.

[8] N. B. Kopnin, F. Taddei, J. P. Pekola, and F. Giazotto, "Influence of photon- assisted tunneling on heat flow in a normal metal-superconductor tunnel junction", Phys. Rev. B, vol. 77, pp. 104517-1 - 104517-10, March 2008.

[9] M. Belogolovskii, M. Grajcar, P. Kúš, A. Plecenik, Š. Beňačka, P. Seidel, "Phase-coherent charge transport in superconducting heterocontacts", Phys. Rev. B, vol. 59, pp. 9617-9626, April 1999.

[10] Ya. M. Blanter, M. Büttiker, "Shot noise in mesoscopic conductors", Phys. Rep., vol. 336, pp. 1-166, September 2000.

[11] M. Belogolovskii, "Phase-breaking effects in superconducting heterostructures", Phys. Rev. B, vol. 67, pp. 100503(R)-1 100503(R)-4, March 2003.

[12] M. B. Hastings, C. J. Olson Reichhardt, and C. Reichhardt, "Ratchet Cellular Automata", Phys. Rev. Lett., vol. 90, pp. 247004 - 1 - 247004 - 4, June 2003. 